\title{
Strong Interactions in Multimode Random Lasers
}

\author{
Hakan E. Türeci ${ }^{1}, \mathrm{Li} \mathrm{Ge}^{2}$, Stefan Rotter $^{2, \dagger}$, A. Douglas Stone ${ }^{2}$ \\ ${ }^{1}$ Institute of Quantum Electronics, ETH Zurich, 8093 Zurich, Switzerland \\ ${ }^{2}$ Department of Applied Physics, P. O. Box 208284, \\ Yale University, New Haven, CT 06520-8284, USA
}

*To whom correspondence should be addressed; E-mail: tureci@phys.ethz.ch.

${ }^{\dagger}$ Presently on leave from Technische Universität Wien, Wiedner Hauptstraße 8-10/136, A-1040 Vienna, Austria, EU

\begin{abstract}
Unlike conventional lasers, diffusive random lasers (DRLs) have no resonator to trap light and no high-Q resonances to support lasing. Due to this lack of sharp resonances the DRL has presented a challenge to conventional laser theory. We present a theory able to treat the DRL rigorously, and provide results on the lasing spectra, internal fields and output intensities of DRLs. Typically DRLs are highly multimode lasers, emitting light at a number of wavelengths. We show that the modal interactions through the gain medium in such lasers are extremely strong and lead to a uniformly spaced frequency spectrum, in agreement with recent experimental observations.
\end{abstract}

Novel laser systems have emerged recently due to modern nanofabrication capabilities (1] 2] 3). The diffusive random laser (DRL), perhaps the most challenging of the new systems, consists of a random aggregate of particles which scatter light and have gain or are embedded in a background medium with gain (2, 4, 5, 6, 7, 8). While light scattering in such a random 
medium can give rise to Anderson localized, high-Q resonances (9. 10), in almost all experiments the localized regime is not reached and the laser "cavity" has no isolated resonances in the absence of gain. Despite the lack of sharp resonances, the laser emission from the more recent DRLs (6, 5, 2) was observed to have the essential properties of conventional lasers: the appearance of coherent emission with line-narrowing above a series of thresholds, and uncorrelated photon statistics far from threshold (11). Earlier work on random lasers (4) 7) did not find isolated narrow lines and was interpreted as incoherent lasing, in which there was "intensity feedback" but not amplitude feedback. Later experiments (2) and recent numerical studies (12) indicate that the lasing involves coherent phase-sensitive feedback in at least some cases. Our work shows that standard coherent multimode lasing is possible even when the linear resonances are much broader than their spacing, raising the question of what determines the emission frequencies of DRLs, since they are not determined by the position of passive cavity resonances. Furthermore, recent experiments on porous GaP DRLs have shown that the frequencies are rather uniformly spaced and stable from pulse to pulse, while the intensities vary substantially $(8)$. We show that this is a result of strong non-linear interactions between lasing modes combined with extreme leakiness, a regime particularly difficult to treat. In any multimode laser, the different modes compete with one another through the gain medium in a complex manner which depends on the spatial distribution of the electric field of each mode. This is particularly severe in the DRL where there are many spatially overlapping modes with similar (very short) lifetimes.

The finesse, $f$, of a resonator is defined as the ratio of the resonance spacing to the resonance width; standard laser theory only addresses lasers with high finesse (weakly open) resonators and cannot be applied to the DRL which has finesse much less than unity. Hence no analytic results have been derived relating to 2D or 3D DRLs and realistic numerical simulations have been limited due to the computational demands. We introduced a formulation of semiclassical 
laser theory in terms of biorthogonal modes, called constant flux (CF) states, which treats lasing media with any degree of outcoupling and includes the effects of non-linear modal interactions to all orders $(13,14)$. We present analytic and numerical results using this approach applied to a DRL.

The simplest model for a laser which captures all of the relevant spatial complexity are the Maxwell-Bloch equations (15, 16), which are three coupled non-linear equations for the electric field, the polarization and the inversion of the gain medium. For stationary multimode lasing, the modes predicted by these equations are determined by the time-independent self-consistent equation (13)

$$
\Psi_{\mu}(\mathbf{x})=\frac{\mathbf{i} \gamma_{\perp}}{\gamma_{\perp}-\mathbf{i}\left(\mathbf{k}_{\mu}-\mathbf{k}_{\mathbf{a}}\right)} \int \mathbf{d x} \mathbf{x}^{\prime} \frac{\mathbf{D}_{\mathbf{0}}(\mathbf{x}) \mathbf{G}\left(\mathbf{x}, \mathbf{x}^{\prime} ; \mathbf{k}_{\mu}\right) \Psi_{\mu}\left(\mathbf{x}^{\prime}\right)}{\epsilon\left(\mathbf{x}^{\prime}\right)\left(\mathbf{1}+\sum_{\nu} \boldsymbol{\Gamma}_{\nu}\left|\Psi_{\nu}\left(\mathbf{x}^{\prime}\right)\right|^{\mathbf{2}}\right)}
$$

where the electric field is given by $e(\boldsymbol{x}, t)=\sum_{\mu} \Psi_{\mu}(\boldsymbol{x}) \mathrm{e}^{-i \Omega_{\mu} t}$. Here the lasing frequencies $\Omega_{\mu}=c k_{\mu}$ and the lasing mode functions $\Psi_{\mu}(\boldsymbol{x})$ are assumed to be unknown (henceforth we set the speed of light $c=1$ and use the wavevector to denote frequency as well). In Eq. $1 k_{a}$ is the atomic frequency, $\gamma_{\perp}$ is the transverse relaxation rate, $\Gamma_{\nu}=\Gamma\left(k_{\nu}\right)$ is the gain profile evaluated at $k_{\nu}, D_{0}(\boldsymbol{x})=D_{0}\left(1+d_{0}(\boldsymbol{x})\right)$ is the pump, which can vary in space, and $\epsilon(\boldsymbol{x})=n^{2}(\boldsymbol{x})$ is the dielectric function of the "resonator". Electric field and pump strength are dimensionless, being measured in units $e_{c}=\hbar \sqrt{\gamma_{\perp} \gamma_{\|}} / 2 g, D_{0 c}=4 \pi k_{a}^{2} g^{2} / \hbar \gamma_{\perp}$, where $\gamma_{\|}$is the longitudinal relaxation rate and $g$ is the dipole matrix element of the gain medium. Note that each lasing mode $\Psi_{\mu}$ depends non-linearly on all of the other lasing modes through the denominator in Eq.1 which represents the "spatial hole-burning" $(15)$ interaction with the other modes. Through this mechanism modes which lase first tend to suppress lasing in other modes, particularly those with which they are correlated in space.

For simplicity we study a two-dimensional DRL and take the gain medium to be a uniform disk of radius $R$, which contains randomly placed nanoparticles with constant index greater 
than unity. The light field in the cavity can be either Transverse Magnetic or Transverse Electric polarized perpendicular to the plane of the disk leading to a scalar equation for its normal component. The integral in Eq. 1 is over the gain region and the kernel $G\left(\boldsymbol{x}, \boldsymbol{x}^{\prime} ; k\right)$ is the Green function of the cavity wave equation with purely outgoing boundary conditions (13). This represents the steady state response of the passive cavity to a monochromatic dipole oscillating with frequency $k$ at $\boldsymbol{x}^{\prime}$. The non-hermitian boundary conditions on the Green function lead to a spectral representation $G\left(\boldsymbol{x}, \boldsymbol{x}^{\prime} ; k\right)$ in terms of dual sets of biorthogonal functions $\varphi_{m}(\boldsymbol{x}, k)$ and $\bar{\varphi}_{m}(\boldsymbol{x}, k)$, termed constant flux (CF) states, with complex eigenvalues, $k_{m}(13)$.

The CF states play the role of the linear cavity resonances within our theory and reduce to the quasi-bound (QB) states within the cavity to a good approximation for a high Q resonator (17). Importantly, the CF states are complete within the cavity and generate a conserved photon flux outside the cavity, unlike the QB states (13); for the extremely leaky "cavity" of a DRL the CF and QB states are significantly different but statistically similar (Fig. 1).

Since the CF basis is complete and conserves flux outside the gain region, it is an appropriate basis for representing arbitrary lasing modes $\Psi_{\mu}(\boldsymbol{x})$ of a DRL. To solve Eq. 1 we expand each mode in terms of CF states: $\Psi_{\mu}(\boldsymbol{x})=\sum_{m=1}^{N_{C F}} a_{m}^{\mu} \varphi_{m}(\boldsymbol{x})$; substituting this expansion into Eq. 1 gives an equation for the complex vector of coefficients $\mathbf{a}^{\mu}=\left(a_{1}^{\mu}, a_{2}^{\mu}, \ldots, a_{N_{C F}}^{\mu}\right)$ which completely determines $\Psi_{\mu}$ :

$$
a_{m}^{\mu}=D_{0} \sum_{n} T_{m n}^{\mu} a_{n}^{\mu}
$$

The non-linear operator $T_{m n}^{\mu}$ is written out explicitly and discussed in the Supporting Online Material (SOM) (18).

This formalism allows us to obtain analytic insight into the question of what determines the frequencies of the DRL. In single-mode lasers, each lasing frequency is a weighted mean of the real part of the cavity resonance frequency and the atomic frequency $(16)$, which for a typical high finesse system is very close to the cavity frequency with a small shift ("pull") towards the 
atomic line. If we denote the "conventional" lasing frequency by $k_{\mu}^{(0)}$, we find from Eq. 2 that for the DRL

$$
k_{\mu} \approx k_{\mu}^{(0)}+k_{\mu}^{(c)}
$$

where $k_{\mu}^{(c)}$ is a collective contribution due to all the other CF states which has no analog in conventional lasers. In our parameter regime $\left(k_{a} R \approx 30\right)$ both the conventional and collective terms are important (although the conventional term is larger) and the lasing frequencies have no simple relationship to the "cavity frequencies". The collective term is random in sign and does not always generate a pull towards line center (Fig. 1). We believe that at larger $k_{a} R$ the collective term will dominate.

In Fig. 2 we plot the intensities associated with the lasing modes of Fig. 1 as a function of pump strength, $D_{0}$, measured by the length of the vector of CF coefficients, $I=\sum_{m=1}^{N_{C F}}\left|a_{m}^{\mu}\right|^{2}$. The behavior is very different from conventional lasers, showing complex non-monotonic and re-entrant behavior in contrast to the linear increase found for uniform edge-emitting lasers (14). Analysis reveals that the complex behavior is due to the strong spatial hole burning interactions in these systems. The inset shows the lasing frequencies associated with the modes as a function of pump; of the eight lasing modes in the interval, there are six which form three pairs nearby in frequency and their behavior is highly correlated. Evaluation of the overlap of the $\mathbf{a}^{\mu}$ vector associated with each pair of modes confirms that not only their frequencies, but also their decomposition into CF states are similar.

Equations 12 imply that modes with similar $\mathbf{a}^{\mu}$ vectors and similar frequencies will compete strongly because this leads to a hole-burning denominator which is spatially correlated with the numerator. However it is not obvious that frequency quasi-degeneracy should be associated with spatial correlation for the DRL. For random lasers with Anderson localization $(9,10)$ the CF states would typically be spatially disjoint, the $T_{m n}(k)$ operator (cf. Eq. S3 in (18)) would be approximately diagonal and there would be no such spatial correlation. In additional cal- 
culations not shown we do find that for larger index nanoparticles, which begin to localize the CF states, the modal interactions are reduced. But for the DRL, $T_{m n}(k)$ is not diagonal and frequency degeneracy would require an eigenvalue degeneracy in this complex pseudo-random matrix (see discussion in SOM (18)). Instead there is eigenvalue repulsion in the complex plane and strong mixing of eigenvectors, resulting in large spatial overlap of quasi-degenerate lasing modes and strong hole-burning interaction. This interaction, in the absence of some special symmetry, tends to suppress one of the two modes leading to well-spaced lasing frequencies as found by Ref. (8). Hence the rigid lasing frequency spectrum could distinguish the DRL from an Anderson localized laser.

This strong interaction of nearly degenerate modes is reflected in a very large increase in the lasing threshold of the second mode of each pair (see Fig. 2 caption and SOM (18)). These interaction effects are strongly non-linear and hence highly sensitive to statistical fluctuations. To illustrate this in Fig. 3 we contrast the intensity behavior of Fig. 2, for which the pump was uniform in space $\left(d_{0}(\mathbf{x})=\mathbf{0}\right)$ to a case in which we have added to the uniform pump a random white noise term $d_{0}(\mathbf{x})$ of standard deviation $\pm 30 \%$ (normalized to the same total power). For this non-uniform pump the third uniform mode (green) now turns on first. It is thus able to suppress the seventh uniform mode (purple) over the entire range of pump powers and acquires almost a factor of three greater intensity at the same average pump power. The intensities of all the interacting pairs show similar high sensitivity to pump profile, while their frequencies remain relatively stable (see inset Fig. 3). Exactly such behavior was observed in shot to shot spectra of DRLs in experiments (8).

Finally, we consider the spatial variation of the electric field in DRLs (Fig. 4). The false color representation of the multi-mode electric field in the laser has a striking property: it is consistently brighter at the edge of the disk than at its center, even though the gain is uniform and there are no special high-Q modes localized near the edge. To demonstrate that this effect 
is not a statistical fluctuation associated with this particular disorder configuration we have averaged the behavior of the entire basis set of CF states over disorder configurations. The result is a non-random average growth of intensity towards the boundary. The origin of this effect is known from earlier work on Distributed Feedback Lasers with weak reflectivity (19); if the single-pass loss is large, then the single-pass gain must also be large in order to lase, leading to a visible non-uniformity of the lasing mode, with growth in the direction of the loss boundary (on average the radial direction for the DRL). As the DRL has fractional finesse (which is not achievable in a one-dimensional geometry) this effect is much larger in these systems and should be observable. Note that this effect means that the electric field fluctuations in DRLs will differ substantially from the random matrix/quantum chaos fluctuations of linear cavity modes (20), first because each mode is a superposition of pseudo-random CF states and second because these CF states themselves are not uniform on average.

The coexistence of gain, non-linear interactions and overlapping resonances (fractional finesse) makes the DRL a more complex and richer system than the widely-studied linear wavechaotic systems. It remains to be seen whether concepts from random matrix theory and semiclassical quantum mechanics (quantum chaos) will prove fruitful in this context. The theory presented here is "ab initio" in the sense that it generates all properties of the lasing states from knowledge of the dielectric function of the host medium and basic parameters of the gain medium; it should be applicable to any novel laser-cavity system.

\section{References and Notes}

1. K. J. Vahala, Nature 424, 839 (2003).

2. H. Cao, J. Phys. A 38, 10497 (2005).

3. O. Painter et al., Science 284, 1819 (1999). 
4. N. M. Lawandy, R. M. Balachandran, A. S. L. Gomes, E. Sauvain, Nature 368, 436 (1994).

5. S. Mujumdar, M. Ricci, R. Torre, D. S. Wiersma, Phys. Rev. Lett. 93, 053903 (2004).

6. H. Cao et al., Phys. Rev. Lett. 82, 2278 (1999).

7. H. Cao, Waves in Random Media 13, R1 (2003).

8. K. L. van der Molen, R. W. Tjerkstra, A. P. Mosk, A. Lagendijk, Phys. Rev. Lett. 98, 143901 (2007).

9. P. Pradhan, N. Kumar, Phys. Rev. B 50, 9644 (1994).

10. V. Milner, A. Z. Genack, Phys. Rev. Lett. 94, 073901 (2005).

11. H. Cao, Y. Ling, J. Y. Xu, C. Q. Cao, P. Kumar, Phys. Rev. Lett. 86, 4524 (2001).

12. C. Vanneste, P. Sebbah, H. Cao, Phys. Rev. Lett. 98, 143902 (2007).

13. H. E. Türeci, A. D. Stone, B. Collier, Phys. Rev. A 74, 043822 (2006).

14. H. E. Türeci, A. D. Stone, L. Ge, Phys. Rev. A 76, 013813 (2007).

15. H. Haken, H. Sauermann, Z. Phys. 173, 261 (1963).

16. Haken, H. Light: Laser Dynamics Vol. 2 (North-Holland Phys. Publishing, Amsterdam, 1985).

17. In a high finesse cavity $\mathrm{QB}$ and $\mathrm{CF}$ states are essentially the same, each lasing mode is associated with one QB state and the lasing frequency takes the value $k_{\mu}^{(0)}$ between the atomic line center and the cavity frequency $q_{1}$ determined by the ratio of $\gamma_{\perp} / \kappa(13)$.

18. Materials and methods are available as supporting material on Science Online and at arXiv:0805.4497. 
19. H. Kogelnik, C. V. Shank, J. Appl. Phys. 43, 2327 (1972).

20. A. Kudrolli, S. Sridhar, A. Pandey, R. Ramaswamy, Phys. Rev. E 49, R11 (1994).

21. This work was supported by NSF grant DMR-0408636, by the Max Kade and W. M. Keck Foundations, and by the Aspen Center for Physics. We thank Robert Tandy, Manabu Machida, Hui Cao, Ad Lagendijk, Patrick Sebbah, Christian Vanneste, and Diederik Wiersma for discussions. 


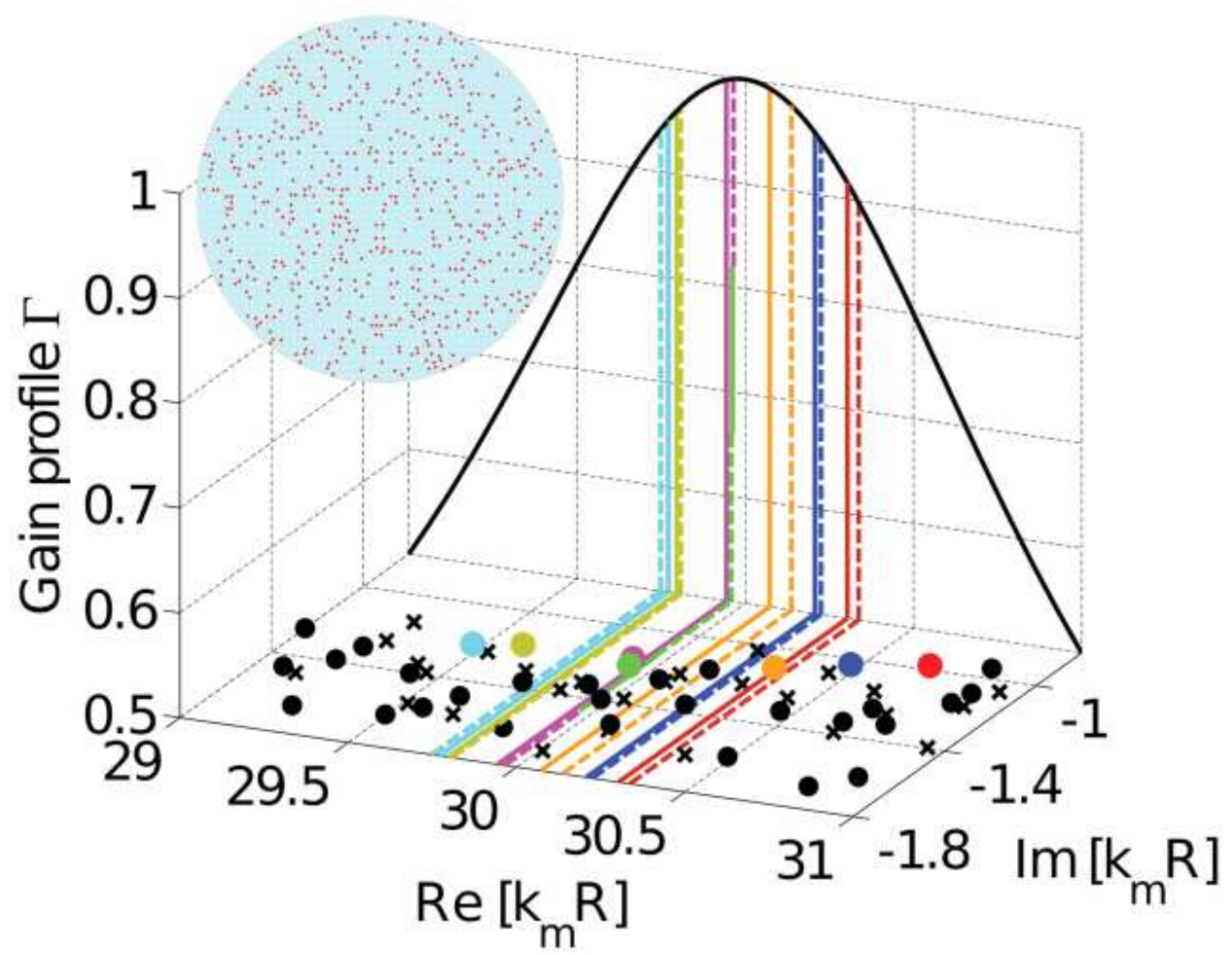

Figure 1: Lasing frequencies of a DRL. Black circles and crosses represent the complex frequencies of the $\mathrm{CF}$ and $\mathrm{QB}$ states, respectively; they are distinct but statistically similar. As their spacing on the real axis is much less than their distance from the axis the system has no isolated linear resonances and the "cavity" has average finesse less than unity $(f \approx .05)$. Solid colored lines represent the actual frequencies $k_{\mu}$ of the lasing modes at pump $D_{0} / D_{0 c}=123.5035$; dashed lines denote the values $k_{\mu}^{(0)}$ arising from the single largest CF state contributing to each mode (the CF frequency is denoted by the corresponding colored circle). The thick black line represents the atomic gain curve $\Gamma(k)$, peaked at the atomic frequency, $k_{a} R=30$. Because the cavity is very leaky the lasing frequencies are strongly pulled to the line center in general, however the collective contribution to the frequency is random in sign. (Inset) Schematic of the configuration of nanoparticle scatterers in the disk-shaped gain region of the DRL. 


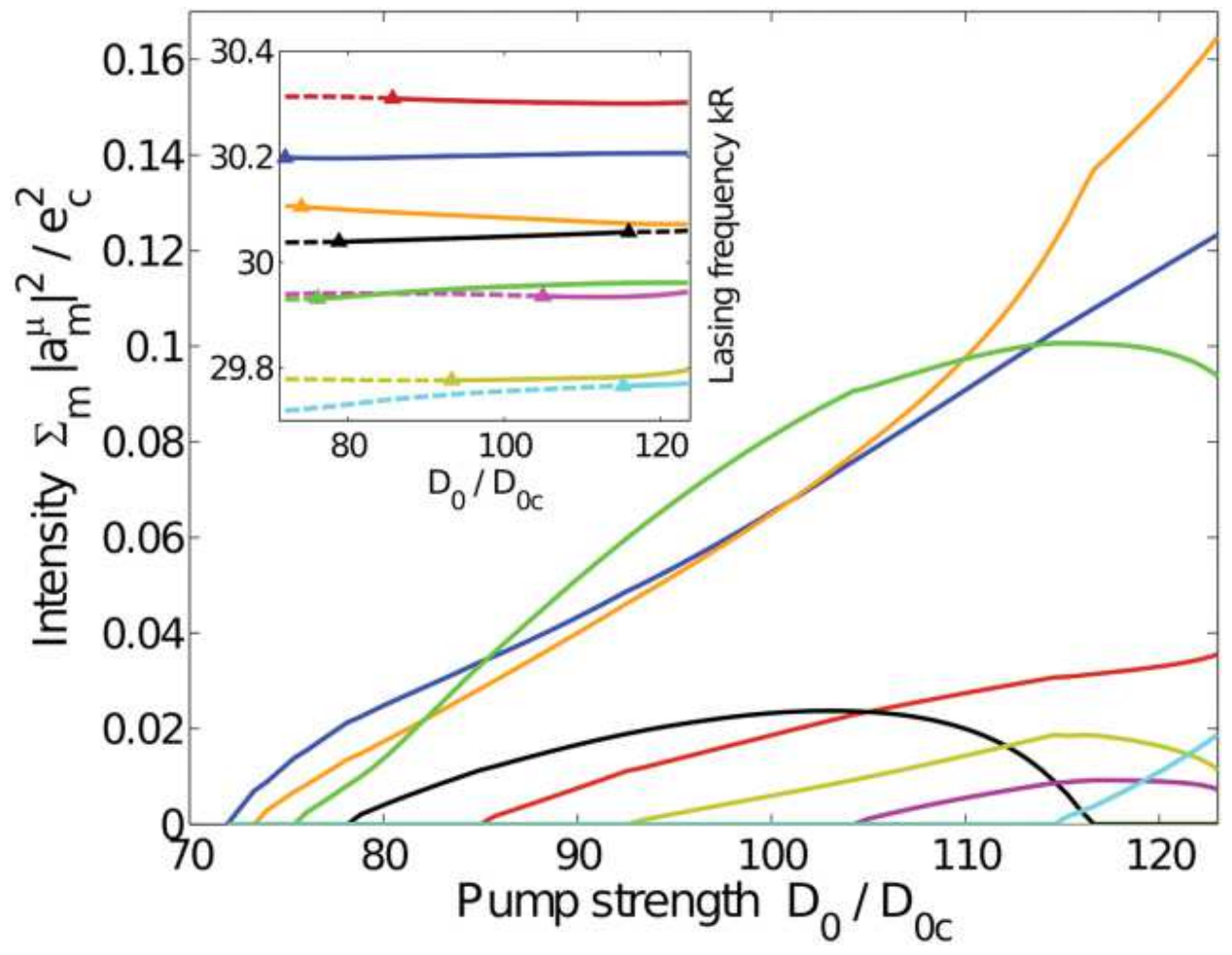

Figure 2: Lasing intensities of a DRL. Modal intensities of a DRL vs. pump $D_{0}$ for the disorder configuration of Fig. 1, illustrating complex non-linear dependence and modal interactions. For example the black and orange modes approach each other in frequency and interact so strongly that the black mode is driven to zero, at which point the orange mode has a kink in its intensity curve. (Inset) The frequencies of each of the eight lasing modes both above (solid) and below (dashed) threshold vs. $D_{0}$. Note that frequencies can cross if one mode is not lasing but not if both are lasing, and modes with nearby frequencies interact strongly so that their intensity curves are highly anti-correlated (see discussion in the text). The interactions increase the lasing thresholds substantially. For example the green and purple modes with $k_{\mu} \approx 29.95$ have non-interacting thresholds $73.4460,75.6919$ respectively, but the hole-burning interaction pushes that of the purple mode up to $D_{0} / D_{0 c} \approx 105$. In addition there would be sixteen modes lasing by $D_{0} / D_{0 c}=93$ in the absence of interactions, compared to the six we find. 


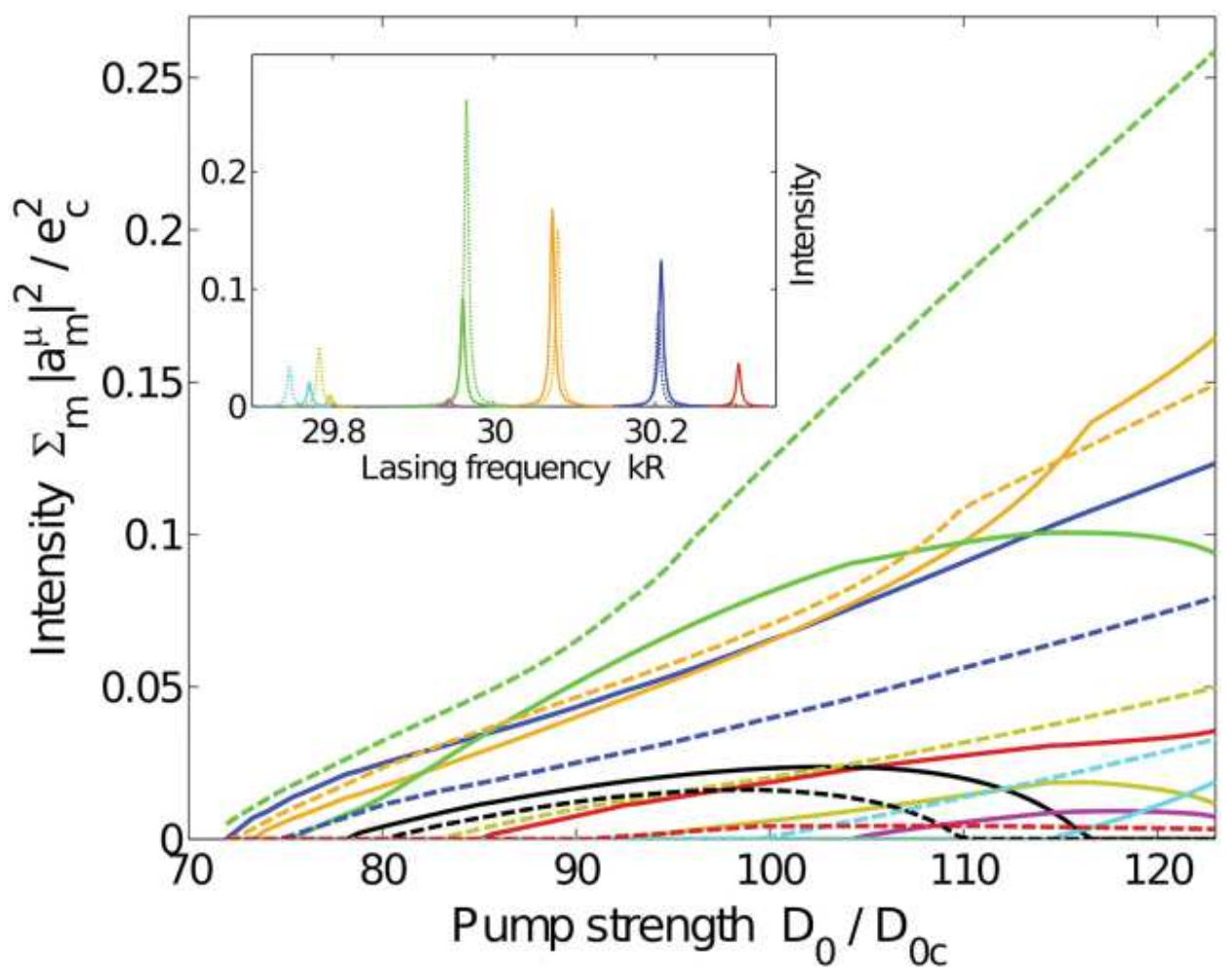

Figure 3: Intensity and frequency fluctuations in a DRL. Comparison of modal intensities of the DRL for the same disorder configuration in the case of uniform pumping (solid lines) and partially non-uniform pumping (dashed lines). The main source of the large intensity fluctuations is the shift in thresholds. This has the largest effect for nearly degenerate mode pairs such as the green and purple modes $\left(k_{\mu} \approx 29.95\right)$. (Inset) lasing spectra at $D_{0} / D_{0 c}=123.5035$ (lines broadened for visibility). Note that the black mode doesn't appear at this pump because it has already been suppressed by the orange mode, and the purple mode only appears for uniform pumping because it never reaches threshold in the non-uniform case. The intensities at this pump can fluctuate by more than a factor of two between the two cases, while the frequencies fluctuate by just a few percent of their average spacing. 


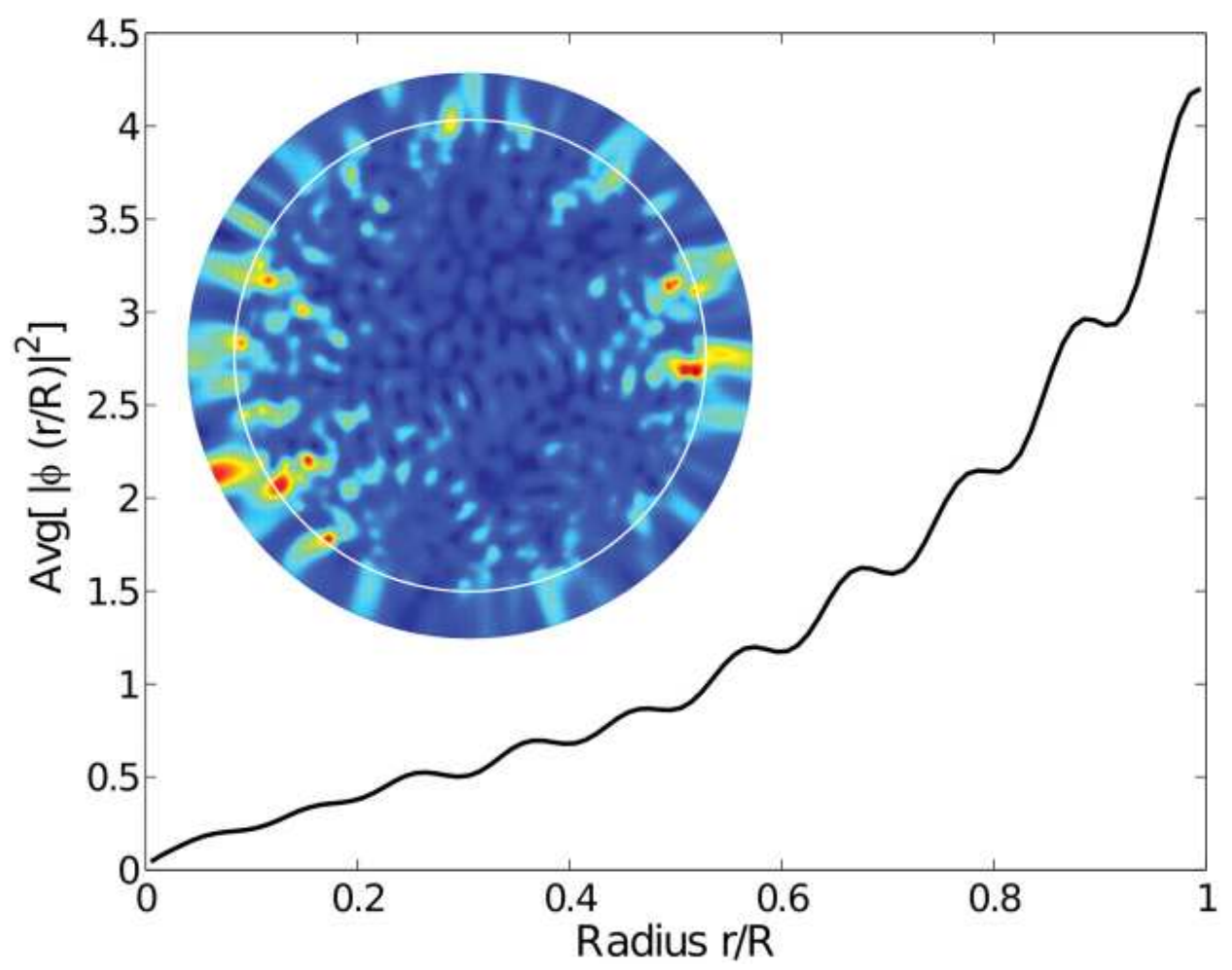

Figure 4: Field distribution of a DRL. Radial intensity of CF states contributing to the lasing modes averaged over 400 disorder configurations. There is a large non-random increase of intensity with radius $r$. (Inset) False color plot of electric field intensity of the seven lasing modes of the DRL of Fig. 1 at pump $D_{0} / D_{0 c}=123.5035$ (white circle is boundary of gain medium). Note brightest regions appear at the edge of the gain medium; this is characteristic of low finesse lasers, but is a particularly large effect in the DRL. 